\title{
Evolution of lithium abundance in Pop I giants
}

\author{
Sushma V. Mallik \\ Indian Institute of Astrophysics, Bangalore 560034, India
}

\begin{abstract}
Recent observations of cool giants have shown that there exists a large range in their lithium abundances even for apparently similar stars. In order to explore whether this large spread could be interpreted in terms of mass, the Li I line at $6707.8 \AA$ has been observed in more than 100 stars and these data have been combined with the already known lithium abundance data in literature. Absolute magnitudes of these stars have been estimated from the Hipparcos data and an attempt has been made to understand the $\mathrm{Li}$ evolution of a star as a function of its mass and the evolutionary status. There are strong evidences for extra-mixing taking place on the red giant branch which explain the unusually low abundances observed.
\end{abstract}

\section{Introduction}

Observations of cool giants have indicated severe depletions in their lithium abundances, far in excess of the predictions of the standard stellar model calculations of e.g. Iben (1967). According to the standard stellar models, the surface dilution of $\mathrm{Li}$ for a $1 M_{\odot}$ star at the tip of the RGB is 28 whereas for a $5 M_{\odot}$ star it is 60 . On the main sequence (MS), while the early $F$ stars and possibly the still hotter stars appear to retain their initial $\mathrm{Li} \log \mathrm{N}(\mathrm{Li})=3.1 \pm 0.2$, it is seen to decrease towards the later spectral types. Consequently, low mass giants $\left(\lesssim 1.5-2.0 M_{\odot}\right)$ are expected to have surface Li much smaller than and up to their maximum predicted value of $1.8 \pm 0.3$ and higher mass giants $\left(\sim 2-5 M_{\odot}\right)$ near their maximum predicted value of $1.5 \pm 0.3$. Observations, however, reveal a large majority of giants having $-1.5 \leq \log \mathrm{N}(\mathrm{Li}) \leq+1.0$. These observations have often been interpreted in terms of the $\mathrm{Li}$ abundance being primarily controlled by the stellar mass. With the Hipparcos data (ESA 1997) it is possible to obtain absolute magnitudes of these stars to a high accuracy and infer their masses on an evolutionary diagram which would give a clue to what progenitors they have evolved from. It is worth exploring then how the Li abundance is linked with mass. With this in mind, a study of the Li I line at $6707.78 \AA$ has been undertaken in a sample of 109 stars. These are described in the next section followed by the interpretation of the observations in Section 3. 


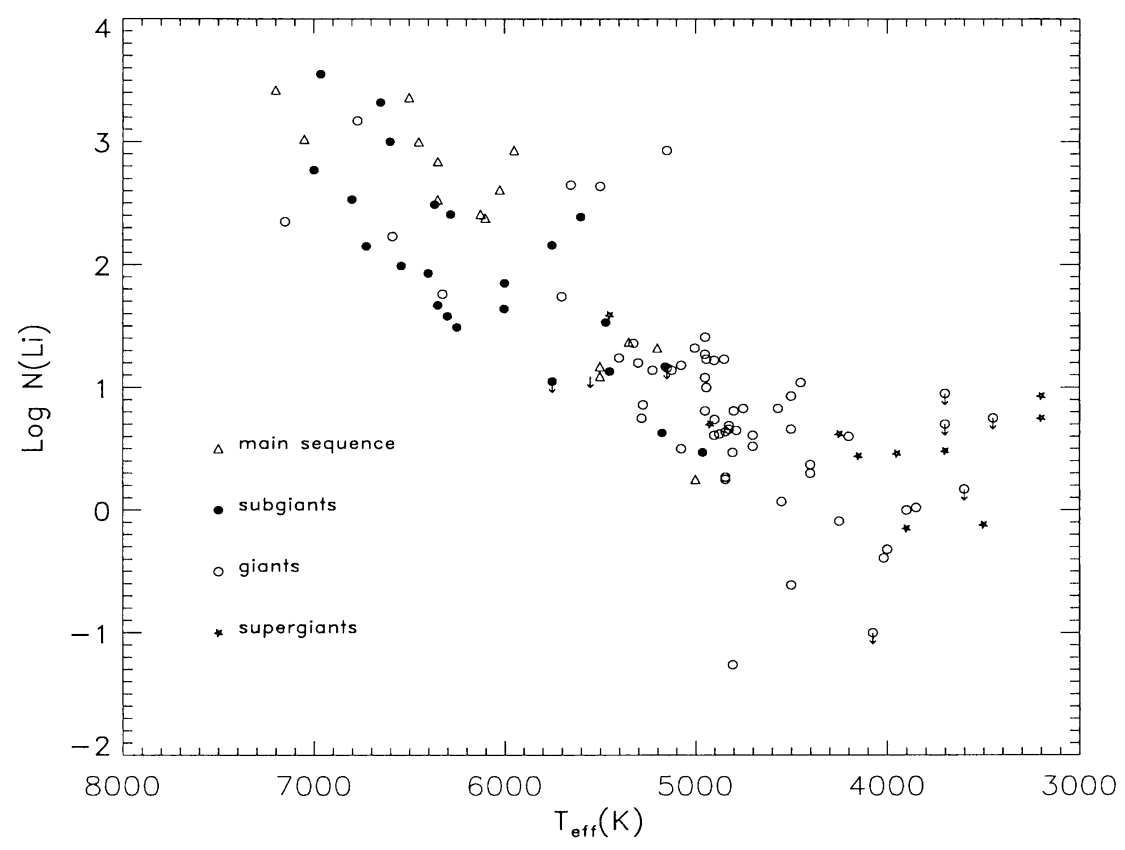

Figure 1. Lithium abundance vs. effective temperature for the 109 observed stars

\section{Observations and Analysis}

109 stars have been sampled from the Bright Star Catalogue (Hoffleit 1982) and the $[\mathrm{Fe} / \mathrm{H}]$ Catalogue of Cayrel de Strobel et al. (1997) spanning spectral types from early $\mathrm{F}$ to late $\mathrm{K}$ mostly comprising of subgiants and giants. A few MS stars and supergiants have also been selected. CCD spectra of these stars have been obtained in the Li I region at a spectral resolution of $\sim 0.35 \AA$ using the coudé echelle spectrograph at the $1 \mathrm{~m}$ telescope at the Vainu Bappu Observatory at Kavalur, India. Standard procedure using the IRAF software has been adopted for reducing the data. With the measured EQWs as the input (after correcting for the Fe I blend at $6707.445 \AA$ ) and an appropriate choice of the models based on the stellar parameters from the grid of model atmospheres due to Gustafsson et al. (1975, upgraded 1992) generated by Luck (1992), Li abundances have been determined using LINES (Sneden 1973, upgraded 1997). The Li abundance depends extremely sensitively, almost exclusively on $T_{\text {eff }} . T_{\text {eff }}$ in the present study has been derived from the recent $T_{\text {eff }}(\mathrm{B}-\mathrm{V})$ calibration of Flower (1996). 


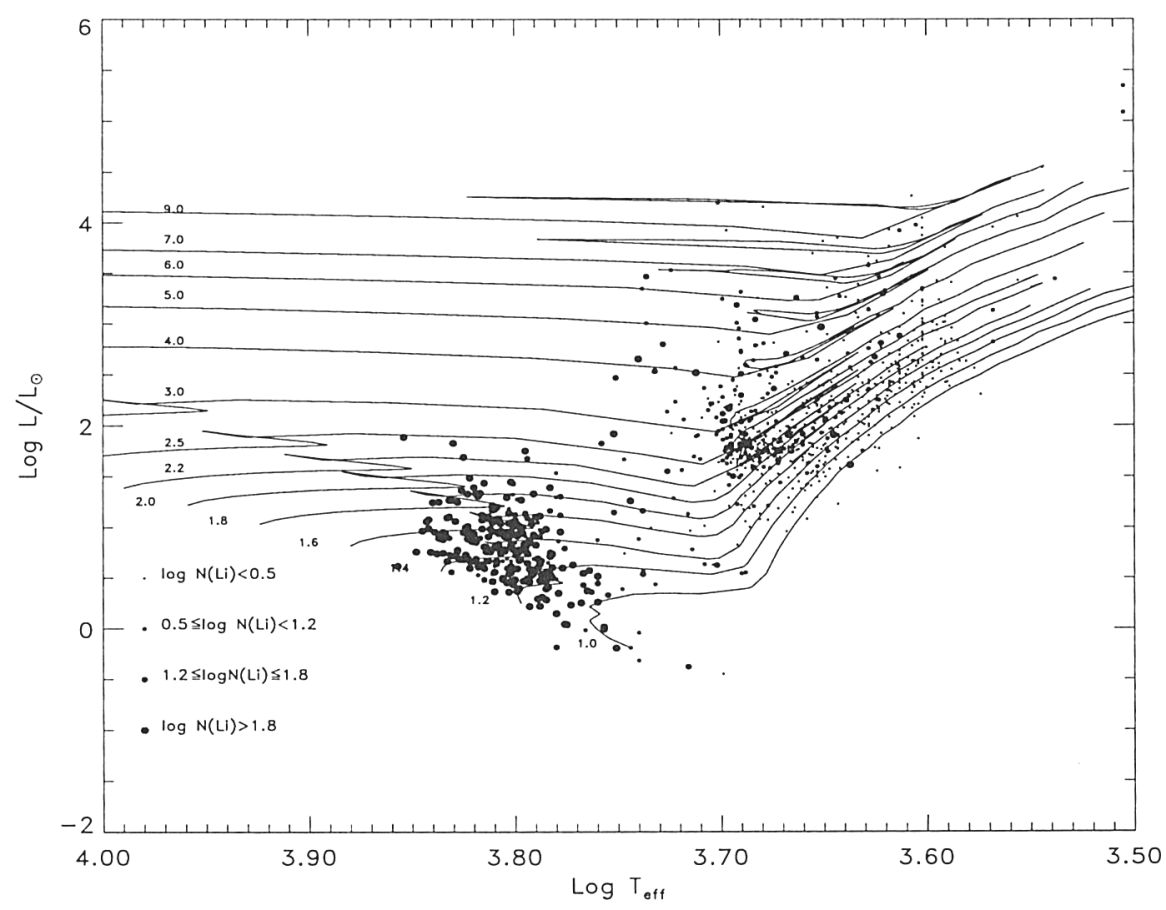

Figure 2. HR diagram of the entire sample of 1024 stars. The log $\mathrm{N}(\mathrm{Li})$ bins are indicated in the key. The theoretical evolutionary tracks are those of Bressan et al. for masses 1.0 to $9.0 M_{\odot}$ for $\mathrm{X}=0.70, \mathrm{Y}=0.28$ and $\mathrm{Z}=0.02$

\section{Interpretation}

\subsection{Evolution of lithium up the red giant branch}

Fig. 1 shows a gradual decline in the Li abundance for the stars observed as a function of $T_{\text {eff }}$ - an evidence of the increasing dilution due to the deepening of the convective envelope. There is a large spread observed at any given $T_{\text {eff }}$ which is perhaps due to the range in masses and evolutionary ages. But by and large, a large number of MS stars and the hotter subgiants have $\log \mathrm{N}(\mathrm{Li})$ close to 3.0, supergiants appear to be heavily depleted and giants lie in between exhibiting a large range in abundances.

We have combined our data with the already known $\mathrm{Li}$ abundance data of another 915 stars from Balachandran (1990):113 MS stars and 49 subgiants and giants; Lèbre et al.(1999):104 subgiants; Pallavicini et al.(1987):6 subgiants; Brown et al.(1989):593 giants and supergiants; Lambert et al.(1980):38 giants and Luck (1997):12 supergiants. From the parallaxes and the apparent visual magnitudes given in the Hipparcos Catalogue, luminosities have been obtained for all these stars using the bolometric corrections from the $T_{e f f}$-B.C. calibration of Flower (1996). The resulting accuracy in $\log \mathrm{L} / L_{\odot}$ is better than 0.08 . There 


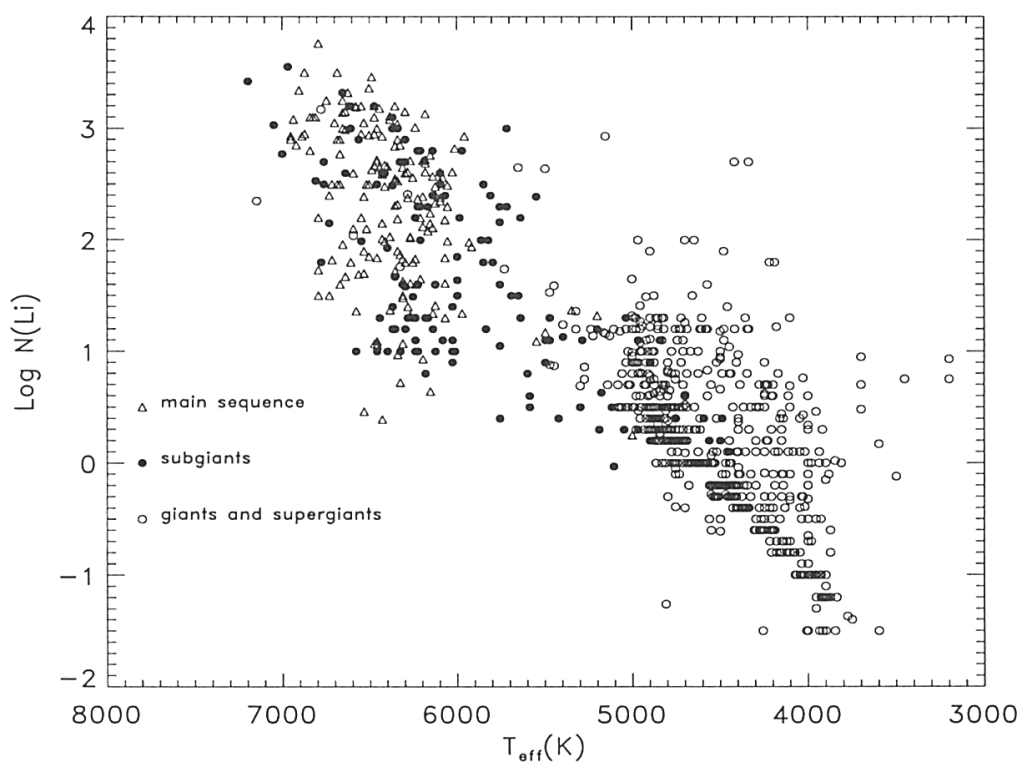

Figure 3. $\log \mathrm{N}(\mathrm{Li})$ vs. $T_{\text {eff }}$ for all the stars plotted in Fig.2

are a few supergiants whose distances exceed 100pc but the rest of the stars are all within $100 \mathrm{pc}$, so the reddening effects are assumed negligible.

Fig.2 shows the positions of the 1024 stars on the HR diagram. Symbols of decreasing size indicate bins of decreasing values of the $\mathrm{Li}$ abundance. Superposed on these stars are the theoretical evolutionary tracks of Bressan et al. (1993) for masses ranging between 1 and $9 M_{\odot}$ with Pop I composition. Based on the location on the HR diagram, one finds that a fraction of subgiants lie very close to the turn-off point or on the MS. Similarly, several giants are actually subgiants and a few of them are supergiants. Although the sample is rather heterogeneous, its largeness aids in understanding the salient features of the evolution of the $\mathrm{Li}$ abundance of a star as a function of its mass and the evolutionary stage. Fig.2 shows a few very obvious trends :

1) Most of the stars around $\log T_{e f f}=3.8$ are either on the MS or evolving off it. Their MS Li has been largely preserved.

2) There is a paucity of stars in the range $3.78>\log T_{\text {eff }}>3.70$ corresponding to the Hertzsprung gap. Depletion as a result of convective dilution is seen in stars as they cross the subgiant branch.

$3)$ The vast majority of the stars in the sample lie at $\log T_{\text {eff }}<3.7\left(T_{\text {eff }} \lesssim 5000 \mathrm{~K}\right)$. These are all red giants and they appear to be severely depleted in $\mathrm{Li}$, particularly those with masses less than $2 M_{\odot}$. Giants more massive than $2 M_{\odot}$ show a somewhat different behaviour. There are several of them which only show the effects of standard post-MS convective dilution. No MS depletion is anyway expected in these massive stars according to the standard stellar models. However, there are an equally large number of giants that have rather low abundances, as low as those in low mass giants. 


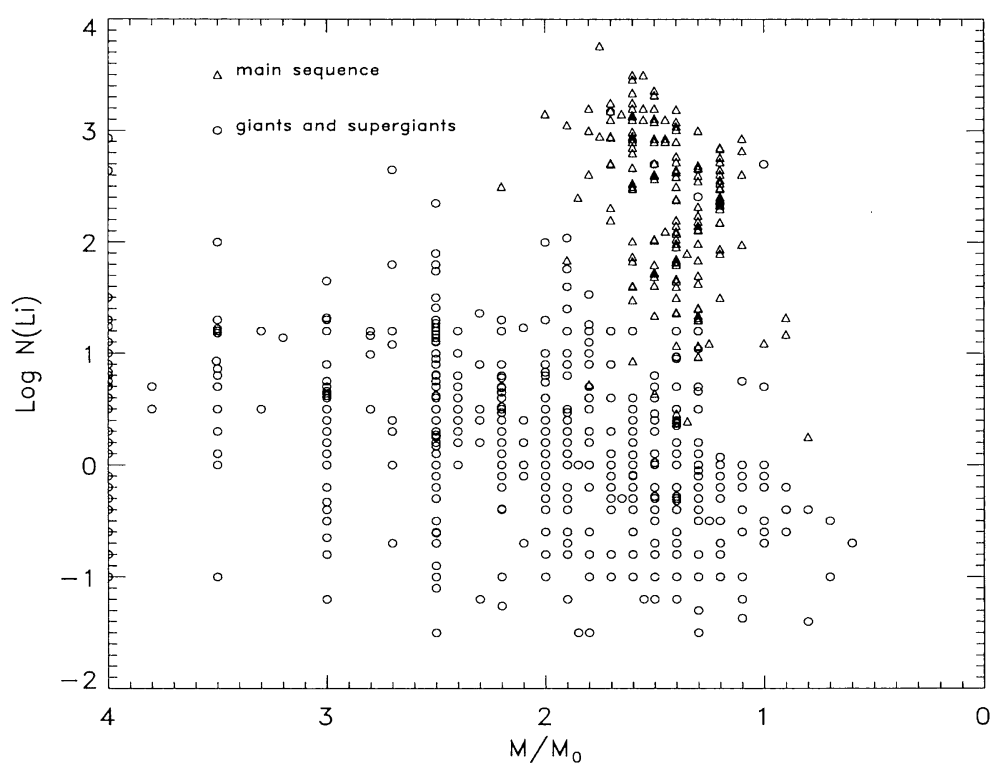

Figure 4. $\log \mathrm{N}(\mathrm{Li})$ vs. $\mathrm{M} / M_{\odot}$ for MS stars and giants of masses less than $4 M_{\odot}$

Some of these trends are seen more clearly in Fig.3 where $\log \mathrm{N}(\mathrm{Li})$ for all the 1024 stars is plotted against $T_{\text {eff }}$. This plot permits us to follow the Li evolution as the star evolves along the subgiant branch and up the red giant branch. The MS stars exhibit a large range in abundances even over their limited range in $T_{\text {eff }}$. A similar spread is seen in subgiants for $T_{\text {eff }} \geq 5400 \mathrm{~K}$. The most remarkable feature is the extremely low values of $\log \mathrm{N}(\mathrm{Li})$ observed in giants in most of them, much lower than what is expected from the standard model predictions after the first dredge up. A large fraction of them have abundances below +0.5 to as low as -1.5 . The Li content goes lower and lower with cooler temperatures. All this implies there must be additional mixing and dilution of $\mathrm{Li}$ on the giant branch. It is clear from the sample of stars in Fig.2 with $T_{\text {eff }}>5000 \mathrm{~K}$ that

1) For subgiants with $\log T_{\text {eff }} \geq 3.73$, dilution due to the deepening of the convective envelope has just about set in and one expects they display a range of abundances basically reflecting the observed spread in the MS stars. In particular, one notices that the Li spread is distinctly apparent between the tracks of 1.4 and $1.2 M_{\odot}$, exactly for stars corresponding to the Boesgaard-Tripicco dip on the MS. So the influence of the MS dip on Li in subgiants is evident.

2) The more evolved stars just to the left of $\log T_{\text {eff }}=3.7$ have undergone deeper mixing. A majority of them have Li already much less than $1.5 \pm 0.3$. There is a strong indication of extra mixing having already taken place even for these stars that are still on the horizontal trail and have not yet begun the ascent up the RGB.

On the other hand, the sample of stars with $T_{\text {eff }}<5000 \mathrm{~K}$ reveals that 


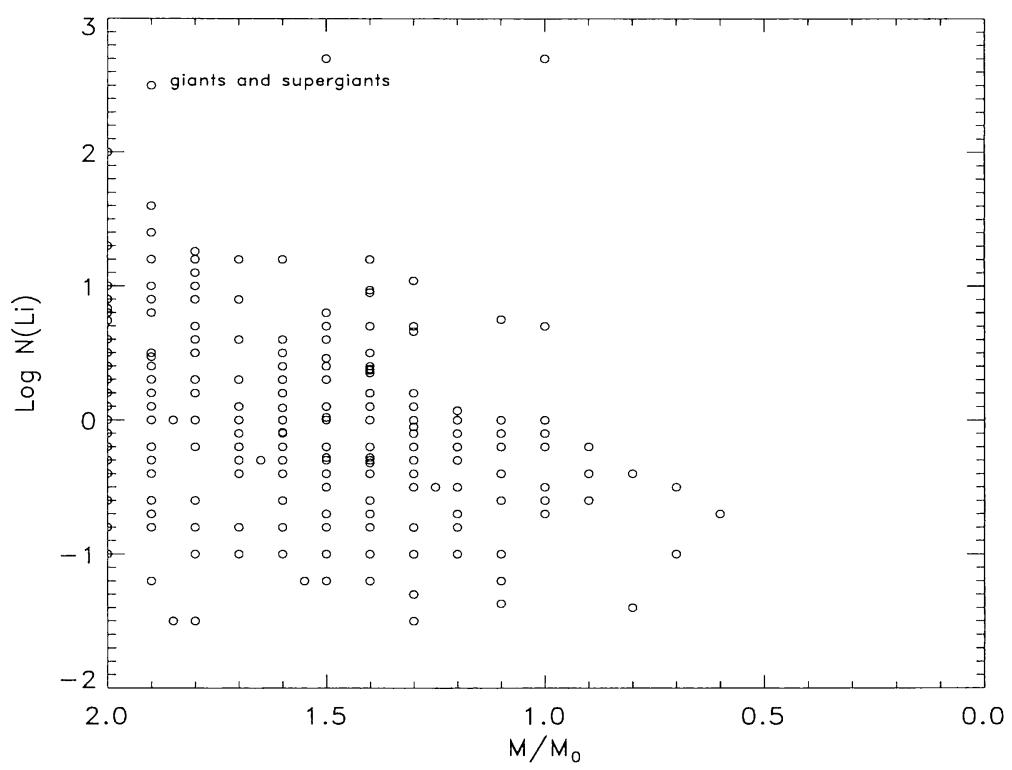

Figure 5. $\log \mathrm{N}(\mathrm{Li})$ vs. $\mathrm{M} / M_{\odot}$ for giants less massive than $2 M_{\odot}$

1) The giants encompass a range of $\mathrm{Li}$ abundances. The scatter for a given mass is a consequence of the dilution effect; stars as they move more to the right on RGB are evidently lower in their Li content. Almost all giants of $\mathrm{M}<2 M_{\odot}$ are heavily depleted independent of the fact that they were in or out of the B-T dip in their MS phase. A very large dilution possibly brings all low mass stars towards a low abundance, whatever their MS Li abundance is. The depletion in the MS phase observed for lower mass stars and the standard convective dilution are surely not sufficient enough to explain these unusually low abundances in them. It is imperative to invoke extra mixing on RGB to explain the observed values. Charbonnel and her collaborators (1994) have computed evolutionary models where they show that the standard convective dilution is essentially complete around $\log \mathrm{L} / L_{\odot}=1.5-2.0$ for the low mass stars, so any further dilution beyond this point is a result of extra mixing on the red giant branch. To be more specific, their calculations reveal that for stars between 1.0 and $1.7 M_{\odot}$ at luminosities between 1.5 and 2.0 respectively, the hydrogen burning shell reaches the chemical discontinuity created by the convective envelope at its maximum extent. The material from the convective envelope has thus access to the $\mathrm{Li}$ burning temperature regions. So there is a process identified in the giant phase that leads to fresh destruction of Li. It is worth noting that the point where $\log \mathrm{N}(\mathrm{Li})$ reaches 0.5 and lower is at a higher luminosity for higher mass. A more systematic detailed study of such data could actually reveal exactly when Li destruction begins for a given mass.

2) Several giants more massive than $2 M_{\odot}$ have also rather low abundances, much lower than the predicted maximum. Their MS progenitors are all earlier than F0, perhaps A and late B. Therefore all observed depletion of Li presumably takes 


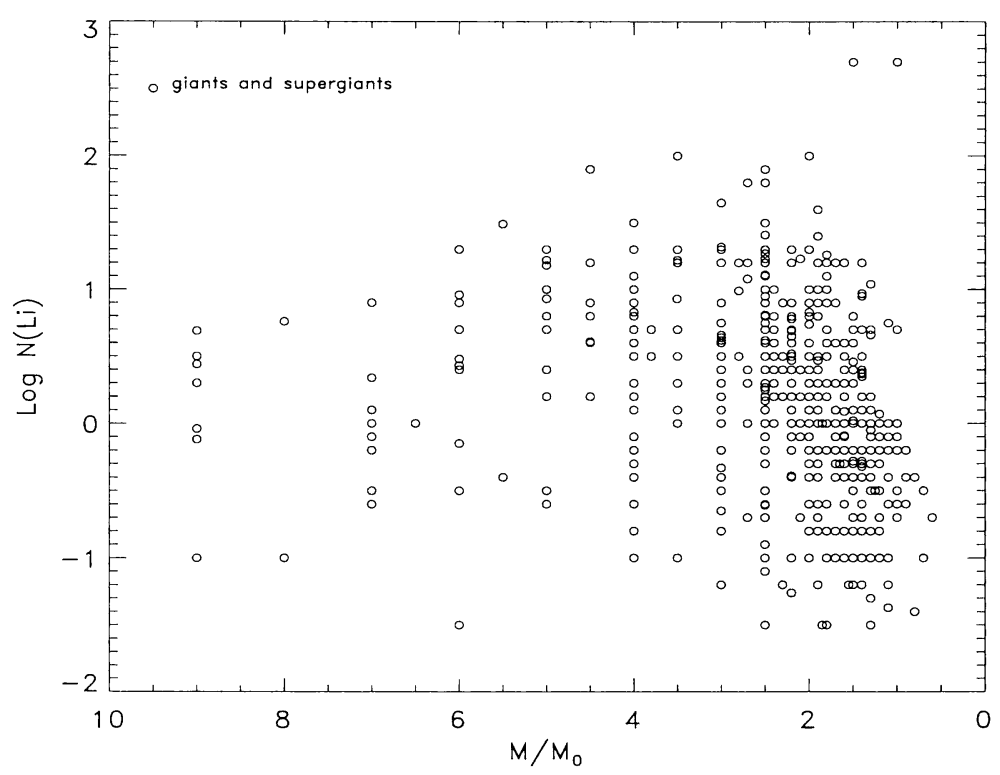

Figure 6. $\log \mathrm{N}(\mathrm{Li})$ vs. $\mathrm{M} / M_{\odot}$ for giants of all masses observed

place only in the post MS phase. One has to invoke non-standard mechanisms even for these more massive stars. Most of the stars more massive than $4 M_{\odot}$ are supergiants and AGB stars and since these are expected to suffer more mass loss than the others, it is likely their Li depletion is further affected. Nevertheless, mass loss and the standard post MS depletion together may not still be adequate to explain the observed low abundances.

\subsection{Lithium vs. Mass}

Masses have been derived for all the stars using the same evolutionary tracks of Bressan et al. Fig. 4 plots $\log \mathrm{N}(\mathrm{Li})$ vs. $\mathrm{M} / M_{\odot}$ for the MS stars and the giants of masses less than $M_{\odot}$. There is a large scatter at all masses but it is hard to overlook a couple of key features. There is a suggestion of a trend among the MS stars of higher Li being associated with a higher mass. A more conspicuous trend seems to exist for giants $\leq 2 M_{\odot}$, roughly over the same range of masses as the MS stars. This is seen better in Fig.5. The upper envelope shows a declining trend of $\log \mathrm{N}(\mathrm{Li})$ for lower masses. One is tempted to interpret this as a consequence of the $\mathrm{Li}$ vs. mass spread present in the progenitors. One notes that the very low values are independent of mass. As pointed out earlier, the depletion is so large that it does not matter how much of it occured on the MS. Giants with masses $2.0 \leq \mathrm{M} / M_{\odot} \leq 6.0$ essentially show a scatter diagram as seen in Fig.6. It could be because they evolve from progenitors which are expected to retain their initial lithium and not show any mass dependence. Lastly, it is curious to note that there is a distinct decline in the average $\mathrm{Li}$ abundance for masses $\geq 6 M_{\odot}$. Most of them are supergiants and AGB stars and are likely to suffer more mass loss than the rest. Most possibly, mass loss combined with 
standard and non-standard mixing causes the lowering of the abundance in these stars.

Acknowledgments. It is a pleasure to acknowledge several stimulating discussions with D.C.V. Mallik

\section{References}

Balachandran, S. 1990, ApJ, 354, 310

Bressan, A. et al. 1993, A\&AS, 100, 647

Brown, J.A. et al. 1989, ApJS, 71, 293

Cayrel de Strobel, G. et al. 1997, A\&AS, 124, 299

Charbonnel, C. 1994, A\&A, 282, 811

ESA 1997, The Hipparcos and Tycho Catalogues, ESA SP-1200

Flower, P.J. 1996, ApJ, 469, 355

Gustafsson, B. et al. 1975, A\&A, 42, 407

Hoffleit, D. 1982, The Bright Star Catalogue, Yale University Observatory

Iben, I. Jr. 1967, ApJ, 147, 624

Lambert, D.L. et al. 1980, ApJ, 235, 114

Lèbre, A. et al. 1999, A\&A, 345, 936

Luck, R.E. 1977, ApJ, 218, 752

Pallavicini, R. et al. 1987, A\&A, 174, 116

Sneden, C.A. 1973, Ph.D. Thesis, The University of Texas, Austin 